Project Report

\title{
Design, Development and Installation of Jordan Subcritical Assembly
}

\author{
Ned Xoubi ${ }^{1,2}$ \\ ${ }^{1}$ Nuclear Energy Group, Center for Strategic Energy Studies, Amman 38111, Jordan \\ ${ }^{2}$ Nuclear Engineering Department, Jordan University of Science and Technology, Irbid 22110, Jordan \\ Correspondence should be addressed to Ned Xoubi; dr.xoubi@yahoo.com
}

Received 31 May 2013; Accepted 13 July 2013

Academic Editor: Keith E. Holbert

Copyright (C) 2013 Ned Xoubi. This is an open access article distributed under the Creative Commons Attribution License, which permits unrestricted use, distribution, and reproduction in any medium, provided the original work is properly cited.

Following its announcement in 2007 to pursue a nuclear power program and in the absence of any nuclear facility essential for the education, training, and research, Jordan decided to build a subcritical reactor as its first nuclear facility. Jordan Subcritical Assembly (JSA) is uranium fueled light water moderated and reflected subcritical reactor driven by a plutonium-beryllium source, and the core consists of $313 \mathrm{LEU}$ fuel rods, loaded into a water-filled vessel in a square lattice of $19.11 \mathrm{~mm}$ pitch. The fuel rods are based on PWR fuel structural pattern type, made of uranium oxide $\left(\mathrm{UO}_{2}\right)$ with $3.4 \mathrm{wt} \%{ }^{235} \mathrm{U}$ enrichment in zirconium alloy (Zr-4) cladding. Design, optimization, and verification were performed using MCNP5 nuclear code; the computed effective multiplication factor $\left(K_{\text {eff }}\right)$ is 0.95923 . The JSA is designed to fulfill the training needs of students and is equipped to perform all of the fundamental experiments required for a typical nuclear engineering university program. This paper presents the design, development, modeling, core analysis, and utilization of Jordan's first nuclear facility and why this simplified low cost reactor presents an attractive choice to fulfill the preliminary experimental needs of nuclear engineering education in developing countries.

\section{Introduction}

The design and construction of the Jordan Subcritical Assembly (JSA) is another step in Jordan's efforts to develop its basic nuclear infrastructure and to introduce nuclear power as part of its energy mix. In 2007 Jordan announced its intention to pursue a nuclear power program [1], joining more than 60 newcomer countries that expressed their interest or declared their intention to develop a nuclear power program for the first time.

Realizing the vital importance of human resource development, Jordan established a nuclear engineering education program at Jordan University of Science and Technology (JUST) [2], with the aim to have graduate qualified engineers that will help in the design, building, operation, and regulation of Jordan's first nuclear power plant.

In the absence of reactors or any other nuclear facility essential for the education, training, and experimental research of its nuclear engineering students, Jordan chose to build a small subcritical reactor as its first nuclear facility.
Located in the Nuclear Engineering Department (NED) at JUST University, the subcritical reactor is the main experimental laboratory which offers students a hands-on experience in nuclear reactor engineering fundamentals $[1,3]$.

From the dawn of the nuclear age subcritical reactors were an essential part of nuclear engineering training and reactor laboratories in advanced nuclear countries worldwide, providing students with practical training in nuclear fission process, in a safe laboratory setting and with minimum exposure to radiation.

\section{Motivation}

Low cost and short construction time in addition to the relative simplicity and accessibility were the main driving force for the design and construction of Jordan Subcritical Assembly (JSA) [4], thus providing timely training for students in the newly established Nuclear Engineering Department, without the burden of large investment or financial 
risk associated with building a research reactor in a small emerging nuclear country.

The reactor simple construction (fuel and moderator) in an open tank makes it one of the most effective tools for educating and training nuclear engineering students, allowing them to change core configuration, work very close to reactor core, observe and see every part of the reactor, and obtain a clear physical picture of the basic features of a reactor in a way that is impossible in power or research reactors $[4,5]$; the JSA reactor hall is shown in Figure 1.

The inherently safe subcritical design enables nuclear engineering students to gain first-hand practical experience on the fundamental physics of the fission process and of a nuclear reactor, with minimal training and radiation exposure and without any risk of criticality accident.

\section{JSA Design}

Jordan Subcritical Assembly (JSA) is uranium fueled light water moderated and reflected small nuclear reactor facility, designed for the purpose of education, training, and experimental research.

JSA is designed to never reach criticality; while being capable of sustaining nuclear chain reaction in the presence of an extraneous neutron source, criticality safety is maintained at all times by its design with a large reactivity margin compared to a critical system and is verified by performing criticality calculations and core analysis [6, 7], making it inherently safe to be operated by students and trainees.

3.1. Design Criteria. The design criteria of Jordan Subcritical Assembly [3] are as follows.

(1) Effective multiplication factor $\left(K_{\text {eff }}\right)$ is $0.94 \sim 0.95$.

(2) Fuel enrichment is 3.4\% wt 3.5\% wt ${ }^{235} \mathrm{U}$.

(3) Neutron emission rate is $1 \times 10^{6} \mathrm{n} / \mathrm{s}$.

3.2. Layout Design. JSA consists of a reactor core, core vessel fuel rods, operation platform, water feed and drainage system, neutron source drive system, I and C system, and a control console [3]; the layout design of JSA main systems is shown in Figure 2.

3.3. Core Design. The reactor core consists of $313 \mathrm{LEU}$ fuel rods, loaded into a water-filled vessel in a square lattice expanding $20 \mathrm{~cm}$ in radius and $55 \mathrm{~cm}$ in height $[1,3]$.

The fuel rods are positioned in the reactor core by two grids that hold the rods in place. The upper grid plate is made of acrylic Plexiglas and the lower grid plate is made of aluminum alloy. The grid is $70 \mathrm{~cm}$ in diameter and is fixed to the reactor vessel with joint poles.

The water in the vessel is high deionized distilled light water, which serves both as a moderator and reflector. When the reactor is to be operated, water is filled using the water feed and discharge system. Water can be drained into the storage tank when the reactor is not in use $[3,8]$.

The reactor vessel is $120 \mathrm{~cm}$ in diameter and $132 \mathrm{~cm}$ in height, enveloping the nuclear core with a water layer of

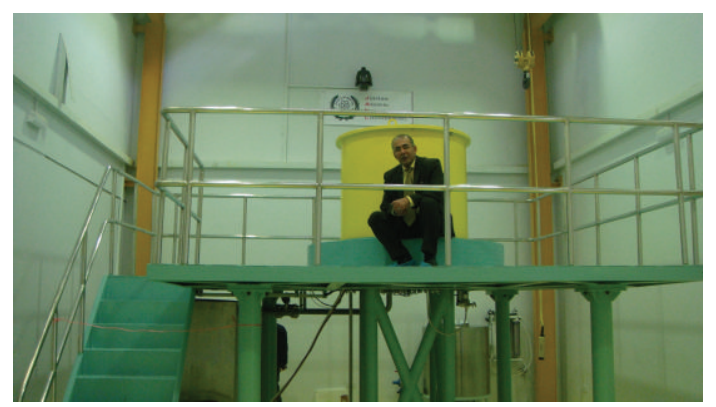

Figure 1: Reactor hall of Jordan Subcritical Assembly (JSA).

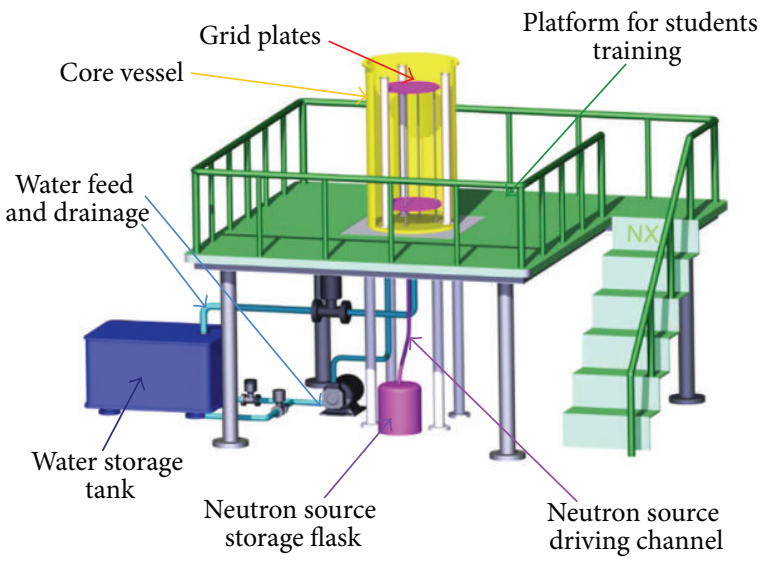

FIgURE 2: Layout design of JSA main systems.

$40 \mathrm{~cm}$ on the sides and $38.5 \mathrm{~cm}$ on top and bottom of the core, as shown in the $x-z$ view of the reactor in Figure 3.

The thick water layer not only serves as a reflector that scatters neutrons back into the reactor core but also as an effective shielding against neutrons leaking out of the system, thus reducing radiation around the reactor; in fact the calculated escape probability of any neutron is found to be less than $0.1 \%$.

3.4. Nuclear Fuel. The design of the JSA fuel rods is based on PWR fuel structural pattern type; essentially it consists of fuel pellets, cladding tube, insulator pellet, pressure spring, upper end plug, and lower end plug, as shown in schematic diagram in Figure 4.

The fuel rod is $550 \mathrm{~mm}$ in height and $10 \mathrm{~mm}$ in diameter, and each rod contains $216.173 \mathrm{~g}$ of uranium in 43 ceramic fuel pellets of uranium oxide $\left(\mathrm{UO}_{2}\right)$ with $3.4 \mathrm{wt} \%{ }^{235} \mathrm{U}$ enrichment, making the active height of fuel $430 \mathrm{~mm}$. Five insulator pellets made of aluminum trioxide are added to the fuel rod: four below the fuel pellets and one above the fuel pellets that is held down by a spring; the fuel rod is plugged at both ends with upper and lower caps $[3,8]$.

The cladding tube is made of zirconium alloy ( $\mathrm{Zr}-4)$, and helium gas is filled in the gap between the fuel pellets and the cladding tube. Fuel specifications and design parameters are listed in Table 1. 


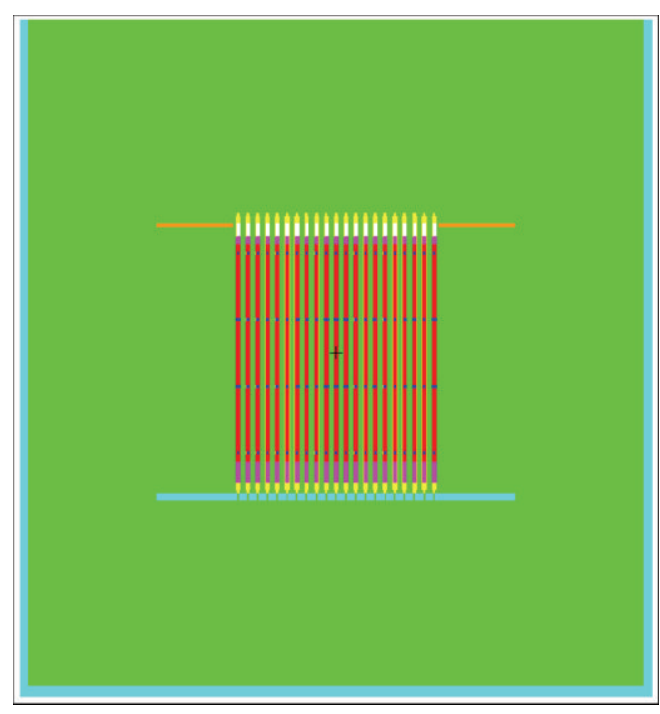

FIGURE 3: MCNP modeling $x-z$ view of JSA reactor, showing the core in the water filled vessel.

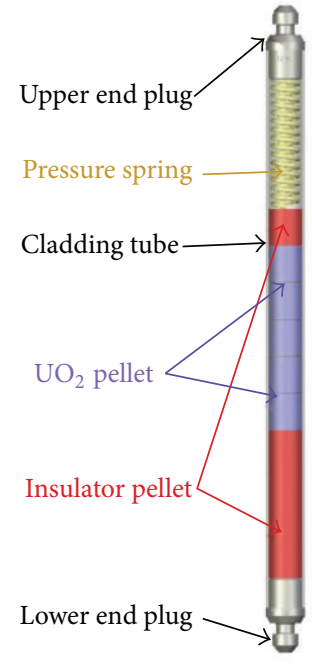

FIgURE 4: Schematic drawing of the JSA fuel rod.

TABLE 1: JSA reactor fuel rod specifications.

\begin{tabular}{lc}
\hline Item & Design parameter \\
\hline Total number of fuel rods & 313 \\
Fuel element height $(\mathrm{mm})$ & 550 \\
Fuel element OD (mm) & 10 \\
Active height (mm) & 430 \\
Number of pellets per element & 43 \\
Fuel material & $\mathrm{UO}_{2}$ \\
${ }^{235} \mathrm{U}$ enrichment (wt\%) & 3.4 \\
Uranium per element (g) & 216.173 \\
Cladding material & $\mathrm{Zr}-4$ \\
\hline
\end{tabular}

3.5. Neutron Source. The JSA is driven by an extraneous neutron source; a plutonium beryllium $(\mathrm{Pu}-\mathrm{Be})$ source was

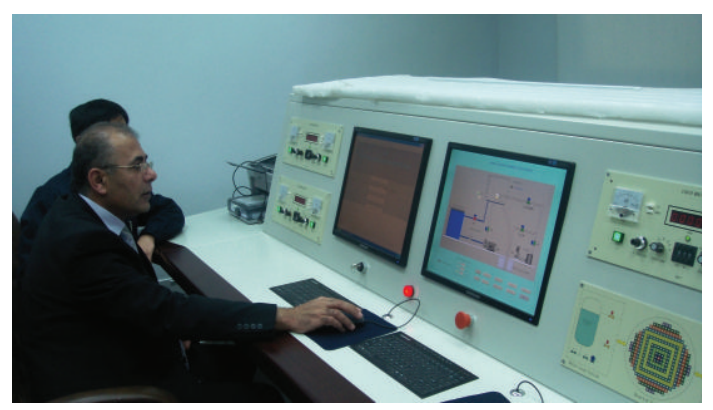

FIGURE 5: JSA reactor control room.

selected because of its long half-life, high neutron yield, and low gamma-ray intensity. The Pu-Be source has an activity of $0.8 \mathrm{Ci}$ and a neutron intensity of $1.1 \times 10^{6} \pm 20 \% \mathrm{n} / \mathrm{s}$.

The source is stored in a shielded underground storage flask, which is filled with borated paraffin wax. For reactor startup, the source is driven into the reactor core through a guide tube by a pneumatic control drive, and to shut down the reactor, the source is driven back to the storage flask.

3.6. Control Room. The JSA reactor is equipped with an advanced digital monitoring and control system that is designed to resemble nuclear reactor control room, as to familiarize students and trainees with reactor control environment and setting; Figure 5 shows the JSA control room.

Reactor startup, shutdown, and operating conditions are monitored and controlled from the control room. Data from detectors and nuclear measurement instruments are displayed and can be saved and printed for normal operation and experiments $[1,8]$.

The advanced system allows for the control, monitoring, display, alarm, and saving of operating parameters of the reactor equipments and systems: neutron source position, electrical and mechanical systems, water fill and drainage, and radiation levels.

The control room is also equipped with a large screen display that monitors the reactor hall through several video cameras at all times; this allows the operator to be in constant contact with the reactor hall, which provides additional safety and security to the reactor. Additionally a small window was also added to allow for visual view of the reactor hall.

The JSA is equipped with a reactor emergency shutdown system: when the shutdown button is pressed the relevant valves open, the neutron source descends into storage cask, the water pump shut down, and the water drain opens, thus keeping the Subcritical Assembly under safe status. The shutdown system is independent of the monitoring system and will shut down the reactor even in case of monitoring system failure.

\section{Modeling and Optimization}

JSA was modeled based on actual design parameters; a threedimensional, full detail, MCNP model denoted as XSR1.0 was developed for this reactor. Criticality and reactor physics 
calculations are performed using the MCNP5 Monte Carlo code $[7,9]$, which has been verified and approved by the nuclear community worldwide. The MCNP-5 code is based on pure transport theory and is accepted by the USA NRC for performing such calculations.

The three-dimensional XSR1.0 simulation model of the JSA uses continuous energy neutron ENDF/B-VII crosssection data libraries and was developed as a nuclear analysis computational tool to calculate nuclear parameters and verify the design [6] using the most recent version of MCNP5 version 1.51 .

The XSR1.0 explicitly models the fuel pellets, clad, helium, fuel elements loading arrangement, grid plates, water moderator/reflector, and the vessel tank; an $x-y$ view of the reactor core at mid-plane, showing fuel rods (red) arrangement surrounded by the water (green) moderator/reflector, is shown in Figure 6.

Likewise, material compositions (atom densities) in the XSR1.0 model were verified by comparison to actual design values, especially for nuclear fuel composition. Ultimately, the total uranium mass of $67660 \mathrm{~g}$ in the XSR1.0 model was found to be in excellent agreement with the reactor actual loaded uranium fuel mass of $67662 \mathrm{~g}$.

4.1. Optimum Moderator to Fuel Ratio. The JSA is designed with the optimum moderator to fuel ratio, so that the reactor is operated in the best moderated condition.

Fuel loading pattern with the optimum moderator-tofuel ratio was obtained using MCNP5 code; the effective multiplication factor as a function of the lattice pitch was calculated and is shown in Figure 7. The optimum value of $19.1 \mathrm{~mm}$ is chosen for the fuel lattice pitch.

4.2. Criticality Calculations. Criticality calculations were performed using Monte Carlo code MCNP5 version 1.51 [9] and the latest nuclear data library ENDF/BVII; the code was installed and tested by running the MCNP criticality validation suite of 31 international benchmark experiments and comparing results $[10,11]$.

The effective multiplication coefficient $K_{\text {eff }}$ [12-14] was calculated by running a total of 550 cycles with 5000 neutrons per cycle, totaling 2,750,310 fission neutron source histories. The final estimated combined (collision/absorption/tracklength) $K_{\text {eff }}$ is $0.95923 \pm 0.00044$.

The average number of fission neutrons produced per neutron absorbed was calculated to be 0.9595 . The majority of neutrons did not cause fission and were absorbed in capture reactions accounting for $60.72 \%$ of neutrons; only $39.2 \%$ of neutrons caused fission producing on average 2.446 neutrons per fission. $93 \%$ of all fissions were caused by thermal neutrons of energy $<0.625 \mathrm{eV}$; criticality calculation results are listed in Table 2 .

\section{Utilization}

JSA is designed mainly as an educational tool for teaching, training, and experimental research of nuclear engineering students and trainees. The simple and safe design provides

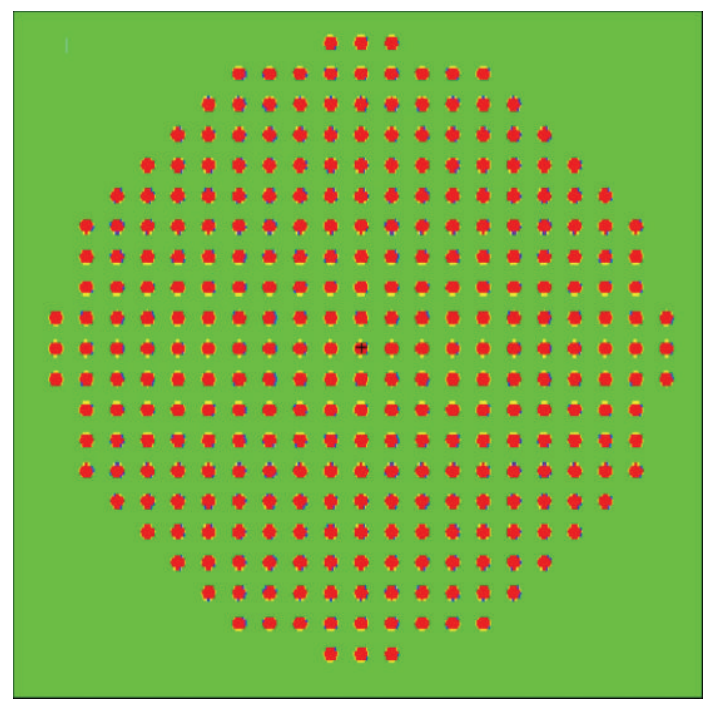

FIGURE 6: MCNP modeling $x-y$ view of the JSA reactor at mid-plane, showing fuel rods (red) arrangement surrounded by water (green) moderator/reflector.

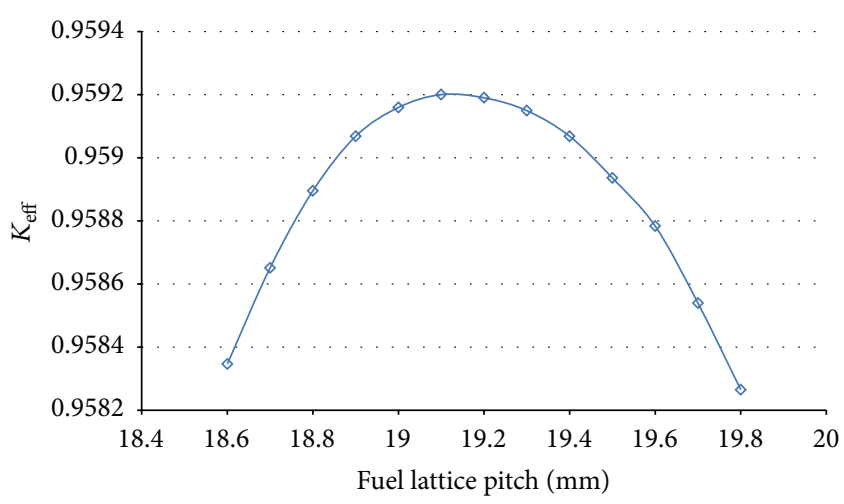

FIGURE 7: The effective multiplication factor $\left(K_{\text {eff }}\right)$ versus fuel pitch.

TABLE 2: Effective multiplication factor $\left(K_{\text {eff }}\right)$.

k-code results (2.5 million particle

histories)

Effective multiplication factor $K_{\text {eff }}$

$0.95923 \pm 0.00044$

Average number of neutrons produced

per fission

2.446

Prompt removal lifetime (sec.)

$9.5452 E-05$

Average number neutrons produced per

neutron absorbed in fuel

1.7087

Fissions caused by thermal neutrons

$(<0.625 \mathrm{eV})$

$93.01 \%$

Neutrons fraction escaping the reactor $\quad 0.08 \%$

Neutrons fraction lost to capture $\quad 60.72 \%$

Neutrons fraction causing fission $\quad 39.19 \%$

students a unique understanding of fundamental aspects of nuclear reactor behavior and hands-on experience in 
determining key reactor parameters $[5,8]$. The JSA will be utilized to perform the following experiments:

(i) approach-to-criticality experiment,

(ii) axial and radial flux distribution,

(iii) absolute flux measurement,

(iv) Rossi- $\alpha$ method,

(v) Feynman- $\alpha$ method,

(vi) source-jerk experiment,

(vii) fuel void effect.

(viii) control rod (poison) effect.

\section{Conclusions}

Subcritical reactors are simplified low-cost nuclear installations that make an excellent choice for small newcomer countries in their pursuit to develop their nuclear infrastructure needed for basic nuclear engineering education, training, and experimental research.

JSA reactor is capable of fulfilling the training needs of students and is equipped to perform all of the fundamental experiments required for the nuclear engineering program including approach-to-criticality, axial and radial flux mapping, absolute flux measurement, Rossi- $\alpha$ method, Feynman- $\alpha$ method, source-jerk experiment, fuel void effect, and control rod (poison) effect.

The reactor effective multiplication factor $\left(K_{\text {eff }}\right)$ of $0.95923 \pm 0.00044$ as calculated by MCNP5-1.5 provides a large reactivity margin $(\Delta \mathrm{K}>0.04)$ compared to a critical system, thus ensuring criticality safety, making the design inherently safe to be operated by students and trainees.

The XSR1.0 three-dimensional simulation MCNP5 model of the JSA reactor is a nuclear analysis computational tool to calculate nuclear parameters which enables experimentalists to carry out calculations and predict reactor parameters for any design change, reactivity effect of experiments, and criticality calculation for new core configuration, before running the actual experiments in the reactor.

\section{Conflict of Interests}

The author declares that, as the Project Manager of Jordan Subcritical Assembly (JSA), he has no conflict of interests.

\section{Acknowledgments}

The author would like to acknowledge the contributions of Dr. Liu Zhenhua and CNNC and CIAE staff to the design and development of JSA and would like to thank JUST University engineers and staff for their cooperation and contributions during project management.

\section{References}

[1] N. Xoubi, "Milestones on Jordan road to nuclear," in Korean Nuclear Society Conference, Pyeongchang, Korea, May 2010.
[2] N. Xoubi, "Development of nuclear engineering education in Jordan," International Atomic Energy Agency, IAEA-CN-153-4P14, IAEA, 2007.

[3] N. Xoubi, "The design \& construction of a subcritical reactor," in Proceedings of the 2nd International Symposium on Nuclear Energy (ISNE '09), pp. 26-28, Amman, Jordan, October 2009.

[4] G. I. Maldonado, N. Xoubi, and Z. Zhao, "Enhancement of a subcritical experimental facility via MCNP simulations," Annals of Nuclear Energy, vol. 35, no. 2, pp. 263-268, 2008.

[5] N. Xoubi, S. Usman, and G. I. Maldonado, "Subcritical reactor experiments at the University of Cincinnati," Transactions of the American Nuclear Society, vol. 90, pp. 420-421, 2004.

[6] N. Xoubi, Z. Zhao, and G. I. Maldonado, "MCNP prediction of measured subcritical reactor parameters," in Nuclear Science Symposium Conference Record (NSSC '05), pp. 874-877, October 2005.

[7] N. Xoubi, R. T. Primm, and G. I. Maldonado, "Neutronic analysis of an advanced fuel design concept for the high flux isotope reactor," Nuclear Science and Engineering, vol. 162, no. 1, pp. 87-97, 2009.

[8] N. Xoubi, “The engineering \& construction of Jordan's first nuclear facility," in International Atomic Energy Agency Consultancy Meeting, pp. 17-18, Vienna, Austria, December 2009.

[9] X-5 MONTE CARLO TEAM, "MCNP—a general Monte Carlo N-particle transport code, version 5," LA-CP-03-0245, LANL, 2003.

[10] F. Brown and B. Kiedrowski, "Recent nuclear criticality safety program technical accomplishments," in ANS Winter Meeting, Washington, DC, USA, 2011, LA-UR-11-06130.

[11] F. Brown, B. Kiedrowski, and J. Bull, "Nuclear criticality safety program technical accomplishments," in ANS Winter Meeting, San Diego, Calif, USA, 2012, LA-UR-12-26115.

[12] C. L. Hollas, C. Goulding, and W. Myers, "Determination of neutron multiplication of subcritical HEU systems using delayed neutrons," Nuclear Instruments and Methods in Physics Research, Section A, vol. 543, no. 2-3, pp. 559-569, 2005.

[13] A. Gandini, "On the multiplication factor and reactivity definitions for subcritical reactor systems," Annals of Nuclear Energy, vol. 29, no. 6, pp. 645-657, 2002.

[14] K. Kobayashi, "Sensitivity analysis of multiplication rate for subcritical systems," Annals of Nuclear Energy, vol. 32, no. 8, pp. 777-794, 2005. 


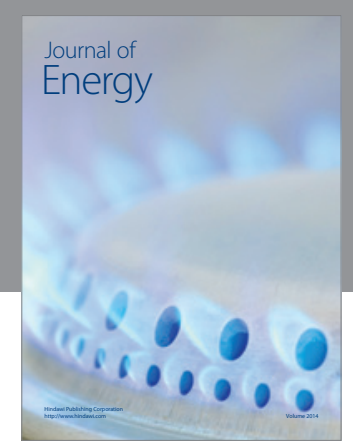

Journal of

Industrial Engineering
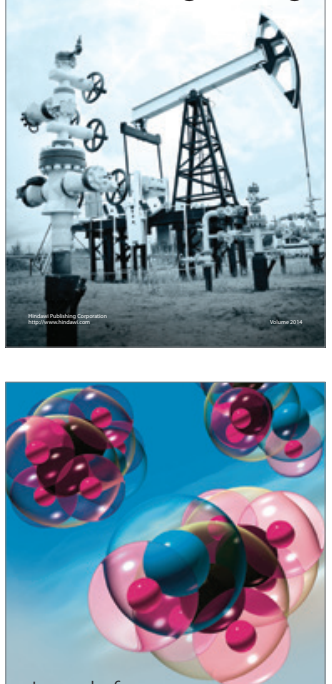

Fuels
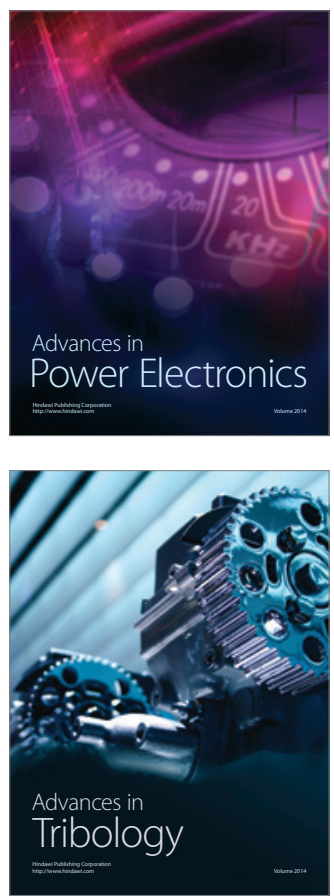

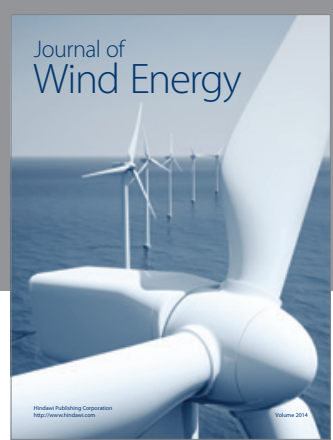

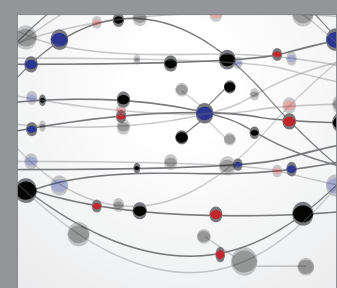

The Scientific World Journal

Submit your manuscripts at http://www.hindawi.com

Journal of

Structures
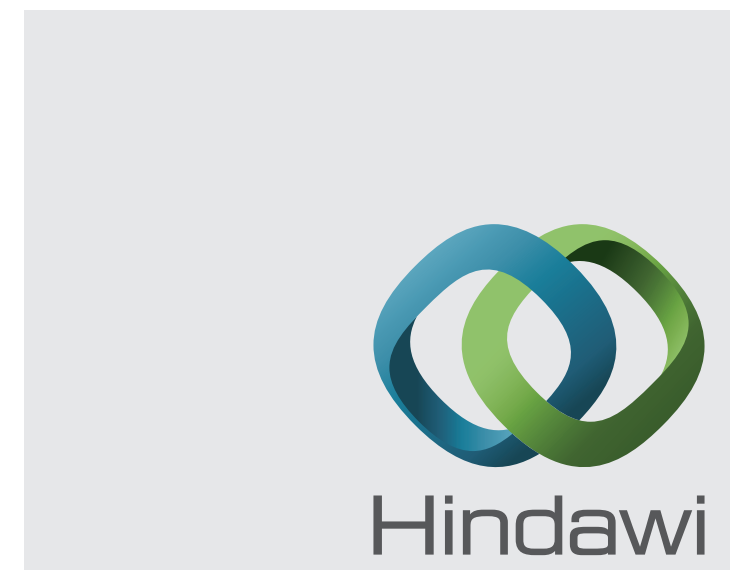

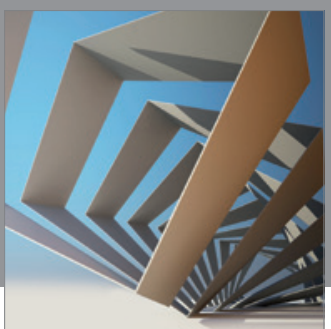

Rotating

Machinery
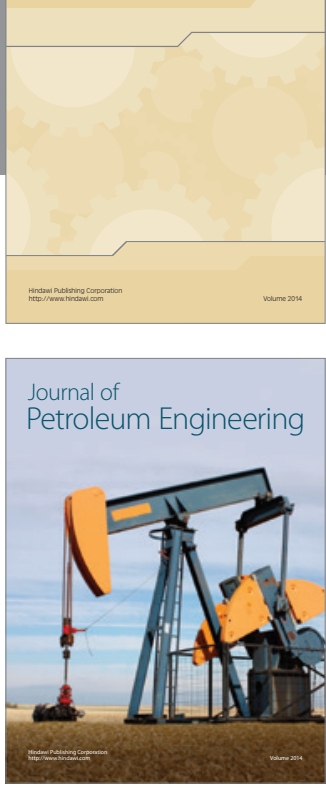

Journal of

Solar Energy
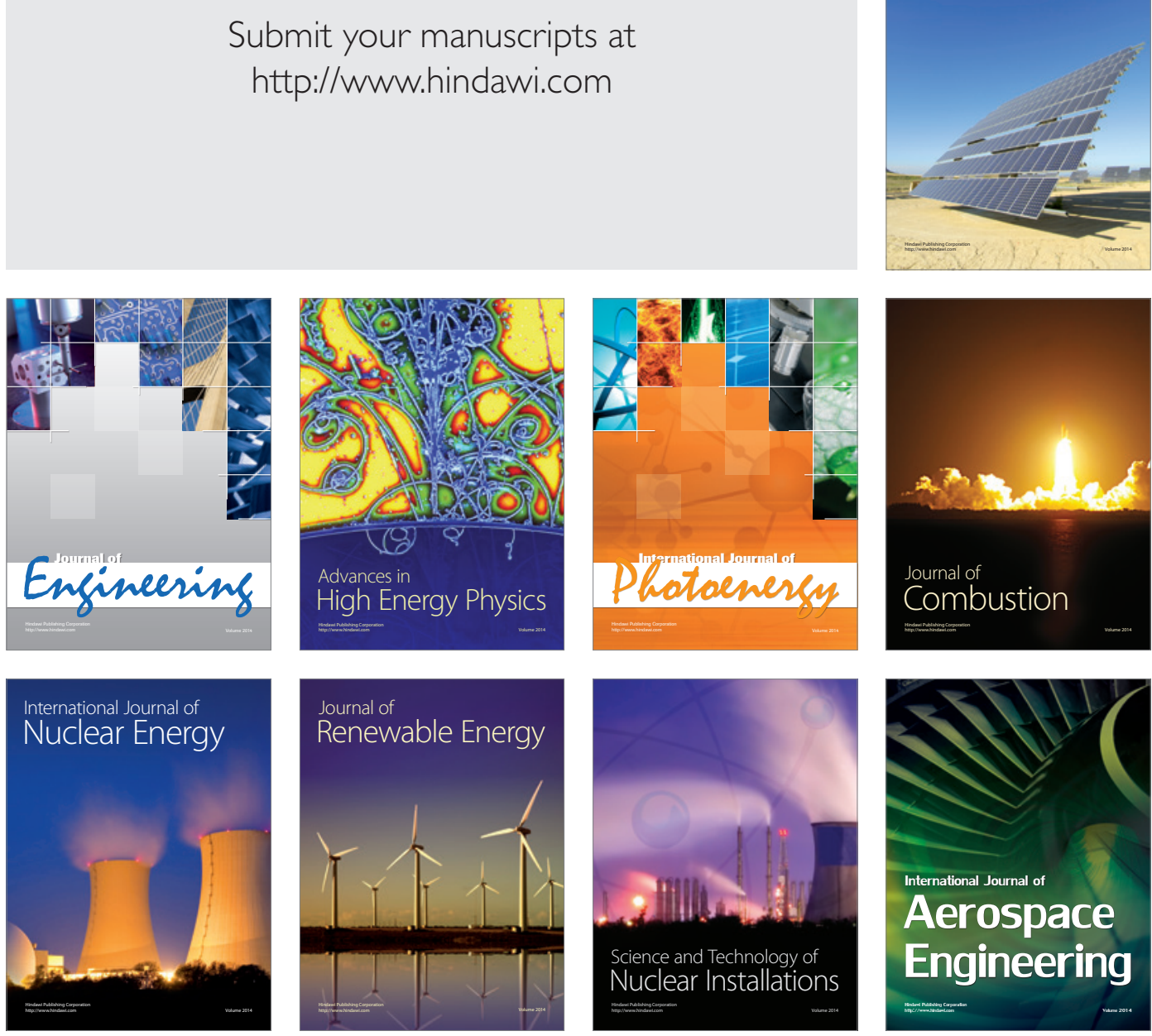